\title{
Cardiology
}

\section{Characteristics of Humoral Regulation of Differentiation of Bone Marrow Monocyte Subpopulations in Patients with Ischemic Cardiomyopathy}

Olga I. Urazova, $\mathrm{PhD}, \mathrm{ScD}^{1,3}$; Svetlana P. Chumakova, $\mathrm{PhD}, \mathrm{ScD}^{1 *}$; Maria V. Vins, $\mathrm{PGS}^{1}$; Elena S. Maynagasheva, PGS ${ }^{1}$; Vladimir M. Shipulin, PhD, ScD ${ }^{1,2}$; Andrey S. Pryahin, $\mathrm{PGS}^{2}$; Vadim S. Poletika, $\mathrm{PGS}^{1}$; Tatyana E. Kononova, $\mathrm{PhD}^{1}$; Yulia V. Kolobovnikova, $\mathrm{PhD}, \mathrm{ScD}^{1}$; Vyacheslav V. Novitskiy, PhD, ScD ${ }^{1,3}$

${ }^{1}$ The Siberian State Medical University

${ }^{2}$ Cardiology Research Institute

${ }^{3}$ Tomsk State University of Control Systems and Radioelectronics

Tomsk, the Russian Federation

\begin{abstract}
Background: Monocytes and macrophages play an important role in atherogenesis and myocardial remodeling. Impaired differentiation of monocyte subpopulations may contribute to ischemic cardiomyopathy (ICMP). The aim of the present research was to study the features of the humoral cytokine-dependent regulation of differentiation of classical, intermediate, non-classical and transitional monocytes in bone marrow (BM) of CHD patients with or without ICMP.

Materials and Methods: Forty-five patients with coronary heart disease (CHD), with and without ICMP (19 and 26 male patients, respectively), were examined. Subpopulations of classical $\left(\mathrm{CD} 14^{++} \mathrm{CD} 16\right)$, intermediate $\left(\mathrm{CD} 14^{++} \mathrm{CD} 16^{+}\right)$, non-classical $\left(\mathrm{CD} 14^{+} \mathrm{CD} 16^{+}\right)$, and transitional $\left(\mathrm{CD} 14^{+} \mathrm{CD} 16^{-}\right)$monocytes in bone marrow $(\mathrm{BM})$ samples were quantified by flow cytometry. Concentrations of IL- $1 \beta$, IL-13, TNF- $\alpha$, IFN- $\gamma$, and M-CSF in BM supernatants and blood plasma were evaluated by ELISA.

Results: Concentrations of all cytokines in the blood and IL-1 $\beta$, IL-13, TNF- $\alpha$, M-CSF in BM supernatants as well as the capacity of M-CSF to activate, and IL-13 to inhibit, differentiation of classical monocytes from intermediate forms-were not dependent on the clinical form of CHD. Monocytopoiesis in ICMP was characterized by elevated BM concentration of IFN- $\gamma$, low M-CSF/IL-13 ratio, and a decreased percentage of classical and intermediate monocytes, accompanied by an increased number of transitional cells in BM, as compared to patients without ICMP.

Conclusion: Excess of IFN- $\gamma$ and low M-CSF/IL-13 ratio in BM were associated with inhibition of differentiation of mature monocyte forms and development of ICMP. (International Journal of Biomedicine. 2019;9(2):91-96.)
\end{abstract}

Key Words: monocytopoiesis $\bullet$ coronary heart disease $\bullet$ cytokines

\section{Introduction}

Ischemic cardiomyopathy (ICMP) remains a major issue in modern cardiology, as pharmacological therapy for this disease is largely ineffective, while surgical treatment often results in a progressive cardiac remodeling. The mortality of

*Corresponding author: Prof. Svetlana P. Chumakova, $P h D, S c D$. Siberian State Medical University, Tomsk, Russia. E-mail:Chumakova_S@mail.ru patients with ICMP within 5 years after hospitalization for heart failure reaches $42.3 \%{ }^{(1,2)}$ ICMP is characterized by diffuse myocardial ischemia (in contrast to focal ischemia found in $\mathrm{CHD}$ ), which results in the dilatation of the heart chambers and cardiomegaly. ${ }^{(3)}$ A key mechanism underlying development of ICMP is the lesion of cardiac microvasculature accompanied by endothelial dysfunction. It is speculated that this process can be also associated with atherosclerotic lesions of small arteries, since patients with ICMP often demonstrate atherosclerosis of the coronary vessels, similar to patients with $\mathrm{CHD}{ }^{(4)}$ 
Pathogenesis of ICMP is not fully understood, and multiple factors are considered to be involved in the underlying cardiac dilatation, including cellular infiltration of the myocardium, apoptotic death of cardyomyocytes, destruction of the interstitial matrix, proliferation of fibroblasts and associated collagen synthesis, and viral infections. ${ }^{(3-6)}$ Among cells present in the cardiac tissue, macrophages are considered to be critically involved in the above mechanisms, as well as in the processes of atherogenesis. ${ }^{(7,8)}$ Macrophages are represented by several subpopulations that induce various processes in damaged tissues. Their precursors, monocytes, also show considerable functional heterogeneity. Thus, there are classic $\mathrm{CD} 14^{++} \mathrm{CD} 16^{-}$cells specialized for phagocytosis, intermediate $\mathrm{CD} 14^{++} \mathrm{CD} 16^{+}$monocytes that are involved in the immune interaction with T-lymphocytes, and non-classical $\mathrm{CD} 14^{+} \mathrm{CD} 16^{+}$cells with high affinity for the endothelium, sometimes referred to as patrolling cells. ${ }^{(7,9)}$ The role and origin of another monocyte subpopulation, transitional $\left(\mathrm{CD} 14^{+} \mathrm{CD} 16^{-}\right)$monocytes, remains to be fully elucidated. They appear to participate in the initiation of immune reactions, and either differentiate from classical monocytes or represent their precursors. ${ }^{(10)}$

A number of studies have demonstrated that in atherosclerosis and heart failure, subpopulation ratios of blood monocytes and tissue macrophages undergo substantial changes. ${ }^{(11-13)}$ In patients with CHD, atherosclerosis of coronary arteries is associated with an increased content of intermediate monocytes and a decreased number of classical subset. ${ }^{(7,11)}$ In contrast, ICMP is characterized by a deficit of non-classical monocytes in the blood, while the number of other monocyte subtypes remains unchanged. ${ }^{(14)}$ This suggests that in ICMP patients, the process of monocyte differentiation in BM might have considerable differences compared to CHD patients without ICMP. Identification of patterns of humoral regulation of monocytopoiesis in ICMP will allow for further elucidation of the pathogenesis of this disease and, possibly, discovery of new treatment strategies. Thus, the aim of the present research was to study the features of the humoral cytokine-dependent regulation of differentiation of classical, intermediate, non-classical and transitional monocytes in BM of CHD patients with or without ICMP.

\section{Materials and Methods}

The study included 45 male CHD patients with stable angina (FC II-IV) and NYHA class II-III.. All patients had a history of acute myocardial infarction and underwent coronary bypass surgery in combination with reconstruction of the left ventricular cavity under artificial blood circulation. CHD patients were divided into two groups: Group 1 included 19 patients (mean age of 52.23 \pm 4.09 ) with ICMP (group ICMP+: LVEF $\leq 40 \%$, acute myocardial infarction or revascularization, $\geq 75 \%$ stenosis of left main coronary artery or proximal left anterior descending artery, or $\geq 75 \%$ stenosis of two or more epicardial vessels) ${ }^{(15)}$; Group 2 included 26 patients (mean age of 59.12 \pm 3.86 ) without ICMP (group ICMP-: LVEF $>40 \%$, acute myocardial infarction or revascularization, $\geq 75 \%$ stenosis of coronary vessels of any localization). At the preoperative stage, both groups received similar pharmacological treatment: antianginal therapy with long-acting nitrates, $\beta 1$ adrenergic receptor blockers, and calcium channel blockers; hemostasis correction using antiaggregants, and correction of lipid metabolism using statins. Premedication and induction of anesthesia in patients from both groups was conducted using sedative and anesthetic agents, narcotic analgesics, and muscle relaxants (diazepam, ketamine, fentanyl, promedola, pipecuronium) in comparable doses.

Exclusion criteria were as follows: a) autoimmune diseases, acute phases of allergic diseases, malignancies, hypoplastic, B12-deficient and folate-deficient anemia, chronic infections (viral hepatitis, syphilis, HIV infection); b) preoperative treatment with iron-containing drugs, erythropoietin or immunosuppressive therapy; c) the occurrence of acute infectious diseases less than 3 weeks before surgery; d) patients' refusal to participate in the study.

For all patients (in ICMP+ and ICMP- groups), peripheral venous blood samples were collected from the median cubital vein immediately before the operation. During the operation, after accessing the heart by median sternotomy and prior to establishing cardiopulmonary bypass, red BM was collected from the sternum incision. In BM samples, the percentages of different monocyte subsets, including classical (CD14++CD16-), intermediate (CD14++CD16+), non-classical (CD14+CD16+), and transitional (CD14+CD16-) monocytes, were determined by flow cytometry using monoclonal CD14FITC and CD16-PE antibodies (BD Biosciences, USA), according to the manufacturer's recommendations. The number of all cells positive for CD14 was considered as 100\%.

Blood plasma and BM supernatants were obtained via centrifugation of collected samples at $200 \mathrm{~g}$ and then stored at $-80^{\circ} \mathrm{C}$. Subsequently, concentrations of cytokines, including IL-1 $\beta$, IL-13, TNF- $\alpha$, IFN- $\gamma$, and M-CSF were evaluated by ELISA according to manufacturer's recommendations (for IL$1 \beta$, TNF- $\alpha$ and IFN- $\gamma$ - Vector-BEST, Novosibirsk, Russia; for IL-13 - eBioscience, Austria; for M-CSF - RayBiotech, US).

The study was approved by the local ethics committee at the Siberian State Medical University (protocol №5046 dated November 28, 2016).

Statistical analysis was performed using the Statistica 6.1 software package (Stat-Soft Inc., USA). The normality of distribution of continuous variables was tested by one-sample Kolmogorov-Smirnov test. Non-normal variables were reported as median (interquartile range [IQR]). Mann-Whitney U test was used to compare means of 2 variables not normally distributed. Spearman's rank correlation coefficient was calculated to measure the strength and direction of the relationship between two variables. A probability value of $P<0.05$ was considered statistically significant.

\section{Results and Discussion}

The content of transitional monocytes in BM of patients with ICMP was higher compared to patients without ICMP, while classical and intermediate subpopulations were observed less frequently (Fig.1). The number of nonclassical monocytes showed a tendency to decrease in ICMP- 
and ICMP+ groups, although the difference did not reach statistical significance. The results obtained were in line with the previously described deficit of this monocyte subtype in CHD, and could indicate an impairment of non-classical monocyte generation in patients with CHD accompanied by ICMP. ${ }^{(14)}$ Non-classical monocytes were shown to perform a scavenging function via elimination of dead cells, pathogens and oxidized lipids from vessel walls. ${ }^{(16)}$ Therefore, it can be speculated that insufficient generation of these monocytes in atherosclerosis patients can predispose to an enhanced fixation of lipids to the endothelium of coronary microvessels and thereby contribute to the development of diffuse myocardial ischemia and ICMP. ${ }^{(14)}$

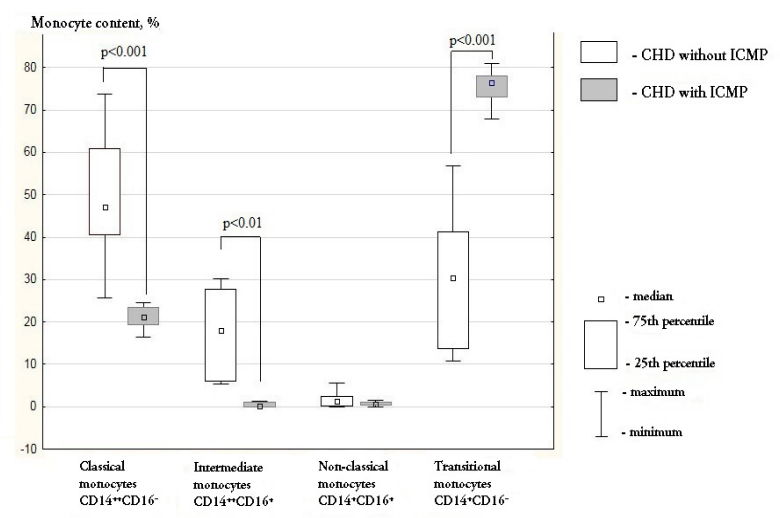

Fig. 1. Subpopulations of bone marrow monocytes in CHD patients with and without ICMP. P-between groups of patients.

In patients without ICMP, classical and intermediate monocytes were the most abundant in BM among studied subpopulations, while in patients with ICMP, transient monocytes were predominant (Fig.1). In both groups, a negative correlation between percentages of transitional and classical cells was observed $(\mathrm{r}=-0.69 ; P<0.01$ and $\mathrm{r}=-0.72$; $P<0.01$ in ICMP+ and ICMP- groups of patients, respectively). Additionally, in patients with ICMP, the number of transitional monocytes inversely correlated with the percentage of intermediate cells $(\mathrm{r}=-0.54 ; P<0.05)$. These results allow us to speculate that transitional monocytes represent the precursors for maturation of classical and intermediate cells, rather than their derivatives. Evidently, this process is impaired in ICMP, and mature forms of monocytes in BM are not sufficiently generated. Differentiation of non-classical monocytes apparently occurs outside BM, since the level of these cells in both groups (ICMP+ and ICMP-) of patients with CHD did not correlate with the percentage of other monocyte subpopulations. Generally, considering that atherosclerotic lesions of coronary arteries are observed both in CHD without ICMP and with ICMP, ${ }^{(4)}$ the differences in the monocyte subpopulation ratio in BM of patients with ICMP relative to patients without ICMP can be viewed as a dysfunction of monocytopoiesis in the setting of atherosclerosis.

Concentrations of IL-1 $\beta$, IL-13, TNF- $\alpha$, and M-CSF in blood plasma and BM supernatants did not differ significantly between the studied groups. At the same time, we observed a multifold increase in the BM level of IFN- $\gamma$ in patients with ICMP compared to patients without ICMP (Table 1). Generally, potential sources of IFN- $\gamma$ include $\mathrm{T}$ helper lymphocytes type 1 (Th1), cytotoxic T lymphocytes, natural killer cells and sometimes regulatory $\mathrm{T}$ cells (Treg), which are present in various tissues, evidently including myeloid tissue. ${ }^{(17)}$ Bansal and colleagues recently demonstrated that development of ICMP is associated with expansion of Treg lymphocytes expressing IFN- $\gamma$ in an experimental murine model. ${ }^{(18)}$ This process could be linked to tissue hypoxia characteristic for ICMP, specifically to the associated production of hypoxia-inducible factors (HIF). During chronic hypoxia, HIF synthesis switches from HIF-1 to HIF-2. As shown previously, a predominance of HIF-2 not only initiates a long-term adaptation to hypoxic condition (by activating angiogenesis, tissue remodeling, etc.), but also contributes to immunosuppression, ${ }^{(19,20)}$ as a deficit of HIF-1 in lymphocytes disinhibits differentiation of Foxp $3^{+}$ regulatory T cells. ${ }^{(21)}$ Collectively, it can be speculated that the dysregulation of monocytopoiesis observed in patients with ICMP could be induced by an excessive medullary production of IFN- $\gamma$, potentially derived from Treg lymphocytes. IFN- $\gamma$ was shown to exert antimitotic and pro-apoptotic effects toward hematopoietic stem cells. ${ }^{(22)}$ It is plausible that IFN- $\gamma$ may also inhibit the differentiation of mature classical and intermediate monocytes, which would explain the observed association of a high medullary concentration of IFN- $\gamma$ with a low content of these cells in BM of patients with ICMP (Fig. 1, Table 1). As for the remaining BM cytokines, in patients with ICMP there was a tendency toward an increase in the content of IL-1 $\beta$, IL-13, TNF- $\alpha$ and a decrease in the concentration of M-CSF relative to their BM levels in patients without ICMP (Table 1).

Table 1.

Concentrations of cytokines in the bone marrow and in the blood of CHD patients with and without ICMP, Me $\left[Q_{1}-Q_{3}\right]$

\begin{tabular}{|c|c|c|c|c|}
\hline \multirow{2}{*}{$\begin{array}{l}\text { Concentrations } \\
\text { of cytokines }\end{array}$} & \multicolumn{2}{|c|}{$\begin{array}{l}\text { CHD patients } \\
\text { without ICMP }\end{array}$} & \multicolumn{2}{|c|}{$\begin{array}{l}\text { CHD patients } \\
\text { with ICMP }\end{array}$} \\
\hline & Bone marrow & Blood & Bone marrow & Blood \\
\hline IL-1 $\beta, \mathrm{pg} / \mathrm{ml}$ & $\begin{array}{c}5.00 \\
{[2.80 ; 24.56]}\end{array}$ & {$\left[\begin{array}{c}2.06 \\
{[0.35 ; 2.74]} \\
P_{1}<0.05\end{array}\right]$} & $\begin{array}{c}8.73 \\
{[4.70 ; 18.45]}\end{array}$ & $\begin{array}{c}2.93 \\
{[0.04 ; 3.65]} \\
P_{1}<0.01\end{array}$ \\
\hline IL-13, pg/ml & $\begin{array}{c}1.00 \\
{[0.80 ; 1.23]}\end{array}$ & $\left.\begin{array}{c}0.60 \\
{[0.41 ; 0.82]}\end{array}\right]$ & $\begin{array}{c}1.22 \\
{[0.80 ; 2.41]}\end{array}$ & $\begin{array}{c}0.82 \\
{[0.40 ; 0.95]}\end{array}$ \\
\hline TNF- $\alpha, p g / m l$ & $\mid \begin{array}{c}10.80 \\
{[9.90 ; 21.84]}\end{array}$ & $\begin{array}{c}1.16 \\
{[0.90 ; 1.82]} \\
P_{1}<0.001 \\
\end{array}$ & $\begin{array}{c}18.06 \\
{[14.15 ; 19.40]}\end{array}$ & {$\left[\begin{array}{c}2.08 \\
{[1.04 ; 3.60]} \\
P_{1}<0.001\end{array}\right.$} \\
\hline IFN- $\gamma, \mathrm{pg} / \mathrm{ml}$ & $\begin{array}{c}0.02 \\
{[0 ; 0.15]}\end{array}$ & 0 & $\begin{array}{c}10.00 \\
{[0.65 ; 18.23]} \\
P_{2}<0.01\end{array}$ & 0 \\
\hline M-CSF, pg/ml & $\begin{array}{c}7.16 \\
{[3.45 ; 16.33]}\end{array}$ & $\begin{array}{c}0.40 \\
{[0.12 ; 2.37]} \\
P_{1}<0.01\end{array}$ & $\begin{array}{c}3.22 \\
{[1.20 ; 8.04]}\end{array}$ & $\begin{array}{c}2.00 \\
{[1.21 ; 3.24]}\end{array}$ \\
\hline $\begin{array}{l}\text { M-CSF/IL-13 } \\
\text { ratio }\end{array}$ & {$\left[\begin{array}{c}9.00 \\
{[2.13 ; 22.09]}\end{array}\right]$} & - & $\begin{array}{c}1.02 \\
{[0.41 ; 2.00]} \\
P_{2}<0.05\end{array}$ & - \\
\hline
\end{tabular}

$P_{1}$ - between cytokine concentration in blood and in bone marrow supernatants; $P_{2}$ - compared to CHD patients without ICMP. 
Interestingly, despite a significant increase in medullary concentration of IFN- $\gamma$ in patients with ICMP, its level was not directly associated with the percentage of individual monocyte subpopulations in BM. Apparently, IFN- $\gamma$ influences monocytopoiesis indirectly, which is further substantiated by the observed correlations between the concentration of M-CSF, a specific inducer of monocytopoiesis, ${ }^{(23)}$ and levels of IFN- $\gamma$ and other medullary cytokines. Concentrations of M-CSF in $\mathrm{BM}$ of patients without ICMP correlated positively to the levels of IL-1 $\beta(\mathrm{r}=0.62 ; P<0.01)$, TNF- $\alpha(\mathrm{r}=0.60 ; P<0.01)$, and IFN- $\gamma(\mathrm{r}=0.72 ; P<0.05)$. In patients with ICMP, however, these associations were not observed, although the M-CSF level correlated to the BM content of IL-13 $(\mathrm{r}=0.72 ; P<0.01)$. Taking into account the data on medullary concentration of studied cytokines and the results of correlation analysis, we can conclude that in CHD patients without ICMP, relatively low medullary levels of IL- $1 \beta$, TNF- $\alpha$, and IFN- $\gamma$ (as compared to $\mathrm{ICMP}+$ group of patients) are associated with hypersecretion of M-CSF (almost a two-fold increase compared to patients with ICMP) (Table 1). Such reciprocal changes could be explained by the mutually potentiating effects of these cytokines on the synthesis of M-CSF. Apparently, a high medullary level of IFN- $\gamma$ in patients with ICMP promotes dissociation of the effects of M-CSF secretion inducers. Thus, an excess of IFN- $\gamma$ appears to indirectly downregulate the differentiation of classical and intermediate monocytes in BM by affecting cytokine-dependent regulation of M-CSF production.

There were no associations between medullary concentrations of cytokines and the percentage of different monocyte subpopulations in BM within ICMP+ and ICMPgroups of patients. Consequently, correlation analysis of these parameters was performed in a pooled sample consisting of all patients with myocardial ischemia, irrespective of the presence or absence of ICMP. In this sample, the medullary concentration of IL-13 correlated positively with the number of transitional monocytes $(\mathrm{r}=0.60 ; P<0.05)$ and negatively with the percentage of classical cells $(\mathrm{r}=-0.53 ; P<0.05)$. In contrast, M-CSF level was negatively associated with the content of transitional monocytes $(\mathrm{r}=-0.52 ; P<0.05)$ and positively associated with the number of classical monocytes $(\mathrm{r}=0.52 ; P<0.05)$. These data, together with the observed correlations among various subpopulations of BM monocytes, allow us to speculate that M-CSF promotes BM differentiation of transitional monocytes into classical cells, while IL-13 inhibits this process.

Despite a putative role of M-CSF and IL-13 in generation of different monocyte subsets, levels of these cytokines in BM did not differ significantly between CHD patients with and without ICMP. However, the medullary content in both ICMP+ and ICMP- groups of patients showed contrasting tendencies. Considering this fact, as well as opposite effects of M-CSF and IL-13 on generation of monocyte subpopulations, as observed in the study, we used M-CSF/IL-13 coefficient as a parameter reflecting the ratio of medullary concentration of cytokines with antagonistic effects on monocytopoiesis. It was found that in CHD patients without ICMP, the M-CSF/ IL-13 ratio was nine times higher compared to patients with ICMP (Table 1). This allows us to consider the M-CSF/IL-
13 coefficient as an indicator parameter for the regulation of monocytopoiesis, as well as an additional (together with IFN- $\gamma$ ) indicator of BM cytokine profile, which differs reliably between CHD patients with and without ICMP.

In order to delineate local and distant cytokine-dependent mechanisms of monocytopoiesis regulation, a comparative analysis of the concentrations of studied cytokines in BM and in the blood was performed. It was shown that in both groups of patients with CHD, concentrations of IL- $1 \beta$ and TNF- $\alpha$ in BM were higher than in the blood, while medullary and plasma levels of IL-13 were comparable (Table 1). In patients without ICMP, the medullary concentration of M-CSF exceeded its plasma level; IFN- $\gamma$ was present in a very low concentration in $\mathrm{BM}$, and was not detected in the blood. In contrast, in patients with ICMP, medullary and plasma levels of M-CSF did not differ, while BM concentration of IFN- $\gamma$ was higher compared to patients without ICMP (Table 1). No statistically significant difference was observed in the plasma concentrations of IL-1 $\beta$, IL-13, TNF- $\alpha$, IFN- $\gamma$, and M-CSF between CHD patients with and without ICMP (Table 1), which indicates the inexpediency of differentiating ICMP+ and ICMP- CHD based on the plasma levels of these cytokines.

To further investigate cytokine-dependent regulation of monocytopoiesis, we searched for correlations between medullary concentration and plasma levels of IL-1 $\beta$, IL13, TNF- $\alpha$, IFN- $\gamma$, and M-CSF in the pooled sample of patients, which proved to be more informative. It was found that the level of IL-13 in BM positively correlated with its concentration in the blood $(\mathrm{r}=0.78, P<0.001)$, while no similar associations were identified for IL- $1 \beta$, TNF- $\alpha$, or M-CSF. Taken together, the results of comparative and correlation analysis of medullary and plasma cytokine levels indicate that IL-13 represent a distant factor of monocytopoiesis regardless of the clinical form of chronic myocardial ischemia, as its concentrations in $\mathrm{BM}$ and blood were comparable and interconnected (Table 1). IL-1 $\beta$ and TNF- $\alpha$, on the other hand, seem to exert a local regulatory effect on monocytopoiesis, since their medullary levels exceeded and were not associated with their plasma concentrations in both groups of patients (Table 1). At the same time, some differences were observed between the studied groups regarding the role of M-CSF and IFN- $\gamma$. Thus, in CHD patients without ICMP, M-CSF apparently functions as a local factor of hemopoiesis (as its medullar concentration was higher than its plasma level), while IFN- $\gamma$ presumably does not have a considerable effect on monocytopoiesis due to an extremely low concentration of the cytokine in BM. In contrast, in patients with ICMP, M-CSF can be considered a distant hemopoietin (as its plasma and bone marrow levels were comparable, Table 1), while IFN- $\gamma$ can be viewed as a local factor of hematopoiesis. Supposedly, in CHD patients with ICMP, bone marrow-derived IFN- $\gamma$ could restrain an activating effect of $\mathrm{M}-\mathrm{CSF}$ on the differentiation of transitional monocytes into classical and intermediate cells. The local influence of IFN- $\gamma$ on monocytopoiesis in ICMP indicates that it is not feasible to control its medullary level through modulation of plasma concentration of the cytokine. At the same time, M-CSF and IL-13, which realize a distant mechanism of monocytopoiesis regulation in patients with 
ICMP, can be considered as potential molecular targets for correction of the monocyte differentiation process via modulating levels of these cytokines in the blood. Demonstrated patterns could open new perspectives on cytokine (or anti-cytokine) therapy for ICMP, thus improving the effectiveness of otherwise largely ineffective treatments for this condition.

Taken together, the current study shows that development of ICMP in patients with CHD is associated with impairment of $\mathrm{BM}$ differentiation of classical and intermediate monocytes, accompanied by local hypersecretion of IFN- $\gamma$ and a decreased ratio of distant hemopoietins M-CSF/IL-13 in BM (as compared with patients without ICMP). Dysregulation of monocytopoiesis in ICMP underlies the suppression of differentiation of mature monocyte subsets, which could affect the subpopulation constitution of blood monocytes and contribute to diffuse atherosclerosis with the development of ICMP. Targeted regulation of these processes through modulation of M-CSF and IL-13 plasma levels could represent a potential treatment modality of therapy for ICMP.

\section{Conclusions:}

In CHD patients with ICMP, percentages of classical and intermediate monocyte subpopulations in BM were lower, while the number of transitional cells and concentration of IFN- $\gamma$ (as a local factor of monocytopoiesis regulation) were higher compared to CHD patients without ICMP. Plasma and medullary levels of IL-1 $\beta$, IL-13, TNF- $\alpha$, and M-CSF in chronic myocardial ischemia did not depend on its clinical form.

M-CSF activates, while IL-13 inhibits, BM differentiation of classical monocytes from transitional cells. A high M-CSF/IL-13 ratio in CHD patients without ICMP was associated with efficient generation of mature forms of monocytes. A decreased M-CSF/IL-13 ratio in CHD patients with ICMP was associated with dysregulation of this process.

IL-13 realizes distant, while IL-1 $\beta$ and TNF- $\alpha$ realize local, mechanisms of monocytopoiesis, irrespective of the clinical form of myocardial ischemia. M-CSF in CHD without ICMP exerts a local regulatory effect on monocyte differentiation. In CHD with ICMP, in contrast, M-CSF can be considered a distant hemopoietin.

\section{Competing Interests}

The authors declare the absence of obvious and potential conflicts of interest related to the publication of the article.

\section{Sources of Funding}

The research was supported by the Russian Foundation for Basic Research (№18-015-00160\18) and Grant Council of the President of the Russian Federation for state support of the leading scientific schools (SS-2690.2018.7).

\section{Acknowledgments}

The authors are grateful to Alexandra G. Pershina $(\mathrm{PhD}$, Senior Researcher of Central Research Laboratory at Siberian
State Medical University) for assistance with flow cytometry, and Prof. Alexander N. Baikov (Honored Worker of Higher Education of the Russian Federation, Head of the Central Research Laboratory at Siberian State Medical University) for the possibility of conducting research on the basis of the Central Research Laboratory.

\section{References}

1. Gutor SS, Kazakov VA, Suhodolo IV, Shipulin VM, Babokin VYe, Ogurkova ON, et al. [Natriuretic peptide and his progenitors as predictors of progressive postoperative left ventricle remodeling in patients with ischemic cardiomyopathy]. Bulletin of Siberian Medicine. 2013; 12(6):25-30. [Article in Russian].

2. Alzahrani T, Tiu J, Panjrath G, Solomon A. The effect of angiotensin-converting enzyme inhibitors on clinical outcomes in patients with ischemic cardiomyopathy and midrange ejection fraction: a post hoc subgroup analysis from the PEACE trial. Ther Adv Cardiovasc Dis. 2018;12(12):351359. doi: 10.1177/1753944718809266.

3. Gavrish AS, Paukov VS. Ischemic cardiomyopathy. Moscow: "GEOTAR-Media"; 2015. [ in Russian].

4. Kaski JC, Crea F, Gersh BJ, Camici PG. Reappraisal of ischemic heart disease. Circulation. 2018;138(14):1463-1480. doi: 10.1161/CIRCULATIONAHA.118.031373.

5. Kozhevnikov ML, Shipulin VM, Sukhodolo IV. [The virus-immune hypothesis for cardiac dilatation]. Ter Arkh. 2017;89(11):79-83. doi: 10.17116/terarkh2017891179-83. [Article in Russian].

6. Xi R, Fan Q, Yan X, Zhang H, Xie H, Gu G, et al. Increased serum interleukin-34 levels are related to the presence and severity of cardiac dysfunction in patients with ischemic cardiomyopathy. Front Physiol. 2018;9:904. doi: 10.3389/ fphys.2018.00904.

7. Rojas J, Salazar J, Martínez MS, Palmar J, Bautista $\mathrm{J}$, Chávez-Castillo $\mathrm{M}$, et al. Macrophage heterogeneity and plasticity: impact of macrophage biomarkers on atherosclerosis. Scientifica (Cairo). 2015;2015:851252. doi: $10.1155 / 2015 / 851252$.

8. Wang LX, Zhang SX, Wu HJ, Rong XL, Guo J. M2b macrophage polarization and its roles in diseases. J Leukoc Biol. 2018 Dec 21. doi: 10.1002/JLB.3RU1018-378RR.

9. Ziegler-Heitbrock L. Monocyte subsets in man and other species. Cell Immunol. 2014; 289(1-2):135-9. doi: 10.1016/j. cellimm.2014.03.019.

10. Ziegler-Heitbrock L, Hofer TPJ. Toward a refined definition of monocyte subsets. Front Immunol. 2013;(4):23. doi:10.3389/fimmu.2013.00023.

11. Fadini GP, Simoni F, Cappellari R, Previato L, Avogaro A. Pro-inflammatory monocyte-macrophage polarization imbalance in human hypercholesterolemia and atherosclerosis. Atherosclerosis. 2014;237(2):805-808. doi: 10.1016/j. atherosclerosis.2014.10.106.

12. Hulsmans M, Sam F, Nahrendorf M. Monocyte and macrophage contributions to cardiac remodeling. J Mol Cell Cardiol. 2016;93:149-155. doi: 10.1016/j.yjmcc.2015.11.015. 13. Shahid F, Lip GYH, Shantsila E. Role of monocytes in heart failure and atrial fibrillation. J Am Heart Assoc. 2018;7(3). pii: e007849. doi: 10.1161/JAHA.117.007849.

14. Azarova DA, Chumakova SP, Urazova OI, Vins MV, Shipulin VM, Pryakhin AS, et al. [Interleukins 4 and 6 as 
factors of modulation of subpopulation composition of blood monocytes in patients with ischemic cardiomyopathy]. Kazan Medical Journal. 2018;99(6):900-905. [Article in Russian]. 15. Felker GM, Shaw LK, O'Connor CM. A standardized definition of ischemic cardiomyopathy for use in clinical research. J Am Coll Cardiol. 2002;39(2):210-8.

16. Dutta $P$, Nahrendorf $M$. Monocytes in myocardial infarction. Arterioscler Thromb Vasc Biol. 2015;35(5):106670. doi: 10.1161/ATVBAHA.114.304652.

17. Urazova OI, Esimova IE, Kononova TE, Zakharova PV, Kolobovnikova YuV, Churina EG. [Molecular mechanisms of the immune response suppression in pulmonary tuberculosis]. Med Immunol. 2017;19(Suppl):206. [Article in Russian].

18. Bansal SS, Ismahil MA, Goel M, Zhou G, Rokosh G, Hamid $\mathrm{T}$, et al. Dysfunctional and proinflammatory regulatory T-lymphocytes are essential for adverse cardiac remodeling in ischemic cardiomyopathy. Circulation. 2019;139(2):206-221. doi: 10.1161/CIRCULATIONAHA.118.036065.
19. Koh MY, Powis G. Passing the baton: the HIF switch. Trends Biochem Sci. 2012;37(9):364-72. doi: 10.1016/j. tibs.2012.06.004.

20. Lin N, Simon MC. Hypoxia-inducible factors: key regulators of myeloid cells during inflammation. J Clin Invest. 2016;126(10):3661-3671. doi: 10.1172/JCI84426.

21. Hsiao HW, Hsu TS, Liu WH, Hsieh WC, Chou TF, Wu YJ, et al. Deltex 1 antagonizes HIF- $1 \alpha$ and sustains the stability of regulatory $\mathrm{T}$ cells in vivo. Nat Commun. 2015;6:6353. doi: $10.1038 /$ ncomms 7353 .

22. Morales-Mantilla DE, King KY. The role of interferongamma in hematopoietic stem cell development, homeostasis, and disease. Curr Stem Cell Rep. 2018;4(3):264-271. doi: 10.1007/s40778-018-0139-3.

23. Jeannin P, Paolini L, Adam C, Delneste Y. The roles of $\mathrm{CSF}$ on the functional polarization of tumor-associated macrophages. FEBS J. 2018;285(4):680-699. doi: 10.1111/ febs. 14343 . 